\title{
Norepinephrine in the Prefrontal Cortex Is Critical for Amphetamine-Induced Reward and Mesoaccumbens Dopamine Release
}

\author{
Rossella Ventura, ${ }^{1,2}$ Simona Cabib, ${ }^{1,2,3}$ Antonio Alcaro, ${ }^{1,2}$ Cristina Orsini, ${ }^{1,2}$ and Stefano Puglisi-Allegra ${ }^{1,2}$ \\ ${ }^{1}$ Dipartimento di Psicologia, Università “La Sapienza," 00185 Rome, Italy, ${ }^{2}$ Istituto di Ricovero e Cura a Carattere Scientifico Fondazione Santa Lucia, 00179 \\ Rome, Italy, and ${ }^{3}$ Istituto di Psicobiologia e Psicofarmacologia, Consiglio Nazionale delle Ricerche, 00137 Rome, Italy
}

Increasing evidence points to a major involvement of cortical areas in addictive mechanisms. Noradrenergic transmission in the medial prefrontal cortex $(\mathrm{mpFC})$ has been shown to affect the motor effects of amphetamine, although there is no evidence of its involvement in the rewarding effects of this psychostimulant.

The present experiments were aimed at investigating the possibility of a selective involvement of prefrontal cortical norepinephrine (NE) in the rewarding-reinforcing effects of amphetamine. To do so, we evaluated the effects of mpFC NE selective depletion in mice of C57BL/6J inbred strain, a background commonly used in molecular approaches that is known to be highly susceptible to the rewarding effects of the psychostimulant. In a first set of experiments, we demonstrated the absence of amphetamine-induced conditioned place preference in mice bearing prefrontal NE depletion. In a second series of experiments, we demonstrated that the same lesion dramatically reduced amphetamine-induced mesoaccumbens dopamine release as measured by intracerebral microdialysis.

These results indicate that noradrenergic prefrontal transmission, by allowing increased dopamine release in the nucleus accumbens induced by amphetamine, is a critical factor for the rewarding-reinforcing effects of this drug.

Key words: prefrontal cortex; norepinephrine; conditioned place preference; reward; nucleus accumbens; dopamine

\section{Introduction}

Drugs of abuse belonging to different classes, although having different primary molecular targets, all increase dopamine (DA) transmission in the nucleus accumbens (NAc) (Di Chiara and Imperato, 1988; Wise and Rompre, 1989; Weiss et al., 1992; Pontieri et al., 1995; Koob et al., 1998; Robbins and Everitt, 1999). In addition, both rewarding-reinforcing and motor-activating effects of addictive drugs are thought to depend on enhanced DA release within the NAc (Wise and Rompre, 1989; Di Chiara, 1995; Koob et al., 1998).

On the other hand, there is increasing evidence of a major involvement of brain norepinephrine (NE) in the behavioral and central effects of drugs of abuse. Thus, it has been shown that mice lacking norepinephrine transporter are behaviorally hypersensitive to psychostimulants (Xu et al., 2000). In contrast, mice lacking $\alpha 1 \mathrm{~b}$-adrenergic receptors are hyposensitive to the behavioral effects of psychostimulants and opioids (Drouin et al., $2002 \mathrm{~b}$ ) and to the enhancing effects of amphetamine (Amph) on dopamine release in the accumbens (Auclair et al., 2002). In addition, prazosin, an $\alpha 1$-adrenergic antagonist, injected either systemically or locally into the prefrontal cortex, was shown to reduce the locomotor hyperactivity induced by D-amphetamine (Snoddy and Tessel, 1985; Dickinson et al., 1988; Blanc et al., 1994; Darracq et al., 1998). Finally, independent studies support

\footnotetext{
Received Aug. 2, 2002; revised Dec. 2, 2002; accepted Dec. 9, 2002.

This work was supported by the Ministero della Ricerca Scientifica e Tecnologica (COFIN 2000-2001) and Ateneo $60 \%(1999-2000)$.

Correspondence should be addressed to Stefano Puglisi-Allegra, Dipartimento di Psicologia, Università “La Sapienza," via dei Marsi 78, 00185 Rome, Italy. E-mail: stefano.puglisi-allegra@uniroma1.it.

Copyright $\odot 2003$ Society for Neuroscience $\quad 0270-6474 / 03 / 231879-07 \$ 15.00 / 0$
}

a role for $\alpha 1$-adrenoreceptors in amphetamine-induced mesoaccumbens DA release (Pan et al., 1996; Darracq et al., 1998; Shi et al., 2000).

Cortical NE might have a critical role in these effects. Indeed, noradrenergic projections are diffuse through the brain cortex, and NE concentrations exceed also those of DA in the medial prefrontal cortex (mpFC). Moreover, a large body of evidence suggests that $\mathrm{mpFC}$ is involved in the mediation of the rewarding and motor-stimulating effects of addictive drugs in rodents (Carter and Pycock, 1980; Bubser and Schmidt, 1990; Tzschentke and Schmidt, 1998a,b). Finally, there is increasing evidence of a major involvement of cortical areas in addictive mechanisms. Indeed, imaging studies in humans suggest that the orbitofrontal cortex, a specific area of the prefrontal cortex, is involved in reinforced behaviors, conditioned responses, and patterns of compulsive drug intake (Volkow and Fowler, 2000).

Amphetamine is a highly addictive psychostimulant that potently stimulates locomotor activity and has strong reinforcingrewarding effects as measured by conditioned place preference (CPP) (Vezina, 1993; Cabib et al., 2000; Robinson and Berridge, 2001). The psychostimulant releases NE more potently than DA (Kuczenski and Segal, 1997; Rothman et al., 2001). However, although a number of recent reports indicate an involvement of mpFC NE in Amph-induced hyperlocomotion and mesoaccumbens DA release (Blanc et al., 1994; Darracq et al., 1998), there are no data on the role of mpFC NE in the rewarding effects of this psychostimulant.

We investigated the effects of mpFC selective NE depletion on rewarding-reinforcing effects of amphetamine as measured by the CPP in mice of the C57BL/6J inbred strain, a background 
commonly used in molecular approaches that is known to be highly susceptible to the stimulating-rewarding effects of amphetamine (Puglisi-Allegra and Cabib, 1997; Cabib et al., 2000). Moreover, we evaluated the effects of prefrontal NE depletion on Amph-induced mesoaccumbens DA release. Indeed, there is convincing evidence for a major role of mesoaccumbens DA release in CPP induced by amphetamine (Carr and White, 1986; Olmstead and Franklin, 1996; Schildein et al., 1998).

\section{Materials and Methods}

Animals

Male mice of the inbred C57BL/6JIco (C57) strain (Charles River, Calco, Italy), 8-9 weeks old at the time of experiments, were housed as described previously (Ventura et al., 2001). Each experimental group consisted of 5-12 animals. All experiments were conducted according to the Italian national law (DLM. 116/92) on the use of animals for research.

\section{Drugs}

D-Amphetamine sulfate, chloral hydrate, 6-hydroxydopamine (6OHDA), and GBR 12909 (GBR) were purchased from Sigma (Milan, Italy). Amph (2.5 mg/kg), chloral hydrate (450 mg/kg), and GBR (15 $\mathrm{mg} / \mathrm{Kg})$ were dissolved in saline $(0.9 \% \mathrm{NaCl})$ and injected intraperitoneally in a volume of $10 \mathrm{ml} / \mathrm{kg}$. 6-OHDA was dissolved in saline containing Na-metabisulphite $(0.1 \mathrm{M})$.

\section{Conditioned place preference}

Behavioral experiments were performed using CPP apparatus (Cabib et al., 1996, 2000). The apparatus comprised two gray Plexiglas chambers $(15 \times 15 \times 20 \mathrm{~cm})$ and a central alley $(15 \times 5 \times 20 \mathrm{~cm})$. Two sliding doors $(4 \times 4 \mathrm{~cm})$ connected the alley to the chambers. In each chamber, two triangular parallelepipeds $(5 \times 5 \times 20 \mathrm{~cm})$ made of black Plexiglas and arranged in different patterns (always covering the same surface of the chamber) were used as conditioned stimuli. The training procedure for place conditioning was described previously by Cabib et al. (1996, 2000). Briefly on day 0 (pretest), mice were free to explore the entire apparatus for $15 \mathrm{~min}$. During the following $9 \mathrm{~d}$ (conditioning phase), mice were confined daily for $40 \mathrm{~min}$ alternatively in one of the two chambers. For each animal, during the conditioning phase, one of the patterns was consistently paired with saline and the other one with either saline or Amph (2.5 mg/kg). Pairings were balanced so that, for one-half of each experimental group, Amph was paired with one of the patterns and one-half of them with the other one. Testing was conducted on day 10 as for the pretest procedure.

Behavioral data were collected and analyzed by "EthoVision" (Noldus Information Technology, Wageningen, The Netherlands), a fully automated video tracking system (Spink et al., 2001). Briefly, a CCD video camera records the experimental system. The signal is then digitized (by a hardware device called a frame grabber) and passed on to the memory of the computer. Later on, digital data are analyzed by means of the EthoVision software to obtain "distance moved" (in millimeters), used as measure of locomotion, and "time spent" (in seconds), used as raw data for preference scores, in each sector of the apparatus by each subject.

\section{Microdialysis}

Animals were anesthetized with chloral hydrate, mounted in a stereotaxic frame (David Kopf Instruments, Tujunga, CA) equipped with a mouse adapter, and implanted unilaterally with a guide cannula (stainless steel; shaft outer diameter, $0.38 \mathrm{~mm}$; Metalant, Stockholm, Sweden,) in the $\mathrm{mpFC}$ or in the NAc. The length of the guide cannula was $1 \mathrm{~mm}$ for $\mathrm{mpFC}$ and $4.5 \mathrm{~mm}$ for NAc. The guide cannula was fixed with epoxy glue, and dental cement was added for additional stabilization. The coordinates from bregma [measured according to the atlas of Franklin and Paxinos (1998)] were as follows (in $\mathrm{mm}$ ): +2.52 anteroposterior and 0.6 lateral for mpFC; and +1.60 anteroposterior and 0.6 lateral for NAc [including mostly the shell subdivision (Franklin and Paxinos, 1998)]. The probe (dialysis membrane length, $2 \mathrm{~mm}$ for $\mathrm{mpFC}$ and $1 \mathrm{~mm}$ for NAc; outer diameter of $0.24 \mathrm{~mm}$; MAB 4 cuprophane microdialysis probe; Metalant) was introduced $24 \mathrm{hr}$ after implantation of the guide cannula. The animals were lightly anesthetized to facilitate manual insertion of the microdialysis probe into the guide cannula. The membranes were tested for in vitro recovery of DA and NE on the day before use to verify recovery.

The microdialysis probe was connected to a CMA/100 pump (Carnegie Medicine, Stockholm, Sweden), through polyethelene-20 tubing and an ultra-low torque dual-channel liquid swivel (model 375/D/22QM; Instech Laboratories, Plymouth Meeting, PA) to allow free movement. Artificial CSF (in mm: $147 \mathrm{NaCl}, 2.2 \mathrm{CaCl}_{2}$, and $4 \mathrm{KCl}$ ) (Pontieri et al., 1995) was pumped through the dialysis probe at a constant flow rate of 2 $\mu \mathrm{l} / \mathrm{min}$. Experiments were performed 22-24 hr after probe placement. Each animal was placed in a circular cage provided with microdialysis equipment (Instech Laboratories) and with home cage bedding on the floor. Dialysis perfusion was started $1 \mathrm{hr}$ later. After the start of the dialysis perfusion, mice were left undisturbed for $\sim 2 \mathrm{hr}$ before collection of baseline samples. Dialysate was collected every $20 \mathrm{~min}$ for $180 \mathrm{~min}$. Three baseline samples before drug treatment were collected. Only data from mice with a correctly placed cannula have been reported. Placements were judged by methylene blue staining. In Figure 1 is represented the location of microdialysis probes in the mpFC and the NAc. Twenty microliters of the dialysate samples were analyzed by HPLC. The remaining $20 \mu \mathrm{l}$ were kept for possible subsequent analysis. Concentrations $(\mathrm{pg} / 20 \mu \mathrm{l})$ were not corrected for probe recovery. The mean concentration of the three samples collected immediately before treatment $(<10 \%$ variation) was taken as basal concentration.

The HPLC system consisted of an Alliance (Waters Corporation, Milford, MA) system and a coulometric detector (model 5200A Coulochem II; ESA, Chelmsford, MA) provided with a conditioning cell (M 5021) and an analytical cell (M 5011). The conditioning cell was set at $400 \mathrm{mV}$, electrode 1 at $200 \mathrm{mV}$, and electrode 2 at $-250 \mathrm{mV}$. A Nova-Pack C18 column $\left(3.9 \times 150 \mathrm{~mm}\right.$; Waters Corporation) maintained at $33^{\circ} \mathrm{C}$ was used. The flow rate was $1.1 \mathrm{ml} / \mathrm{min}$. The mobile phase was as described previously (Westerink et al., 1998). The detection limit of the assay was $0.1 \mathrm{pg}$.

\section{NE depletion in the mpFC}

Anesthesia and surgical set are described in the preceding paragraph. Animals were injected with GBR $(15 \mathrm{mg} / \mathrm{kg}) 30 \mathrm{~min}$ before 6-OHDA microinjection to protect dopaminergic neurons. Bilateral injection of 6-OHDA $(1.5 \mu \mathrm{g} / 0.1 \mu \mathrm{l} / 2 \mathrm{~min}$ for each side), was made into the mpFC [coordinates: +2.52 anteroposterior, \pm 0.6 lateral; -2.0 ventral with respect to bregma (Franklin and Paxinos, 1998)], through a stainless steel cannula (0.15 mm outer diameter; UIMED, Lausanne, Switzerland),

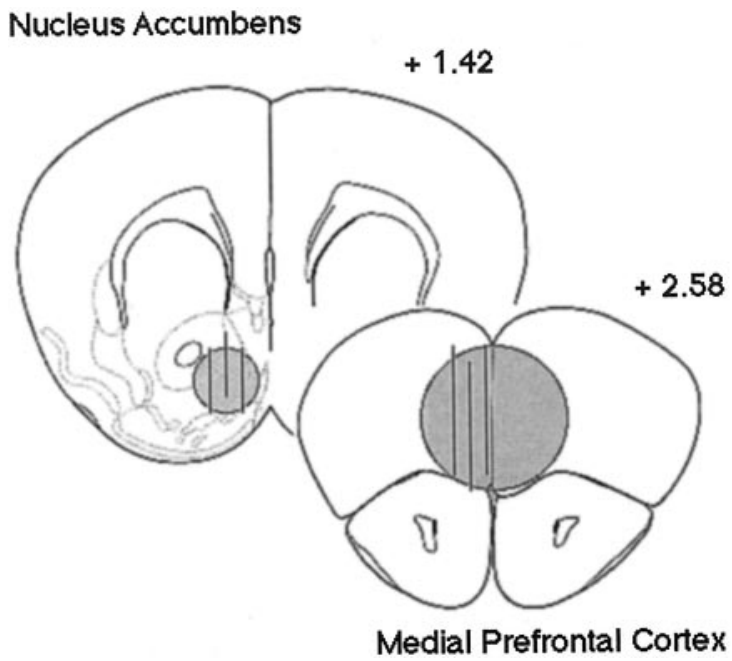

Figure 1. Location of microdialysis probes in the medial prefrontal cortex and the nucleus accumbens. Silhouettes of probe tracks were drawn onto representative sections of the mouse brain and the range of implantation sites. Circles represent the areas punched for tissue analysis. For details, see Materials and Methods. The numbers indicate millimeters rostral to bregma according to Franklin and Paxinos (1998). 
connected to a $1 \mu \mathrm{l}$ syringe by a polyethylene tube and driven by a CMA/100 pump. The cannula was left in place for an additional $2 \mathrm{~min}$ after the end of the infusion. Sham animals (Sham) were subjected to the same treatment but received intracerebral vehicle. Animals were used for microdialysis or behavioral experiments $7 \mathrm{~d}$ after surgery.

$\mathrm{NE}$ and DA tissue levels in the mpFC as well as in the NAc were assessed as described previously (Ventura et al., 2001) to evaluate the amount and the extent of depletion. The brain was fixed vertically on the freeze plate of a freezing microtome. Punches of both hemispheres were obtained from brain slices (coronal sections) no thicker than $300 \mu \mathrm{m}$. Stainless steel tubes of 0.8 (NAc) or $2.3 \mathrm{~mm}(\mathrm{mpFC})$ inner diameter were used. The coordinates were measured according to the atlas of Franklin and Paxinos (1998) as follows (coronal sections as mm from bregma): mpFC, two slices from 2.96 to 2.34 ; NAc, three slices from 1.70 to 0.98 . In Figure 1 is represented punching localization. The punches were stored in liquid nitrogen until the day of analysis.

DA and NE were determined simultaneously using a reverse-phase HPLC procedure coupled with Coulochem electrochemical detection. On the day of the analysis, frozen samples were weighed and homogenized in $0.1 \mathrm{~N} \mathrm{HClO}_{4}$ containing $6 \mathrm{~mm} \mathrm{Na}$-metabisulphite and $1 \mathrm{~mm}$ EDTA. The homogenates were centrifuged at $10,000 \times g$ for $20 \mathrm{~min}$ at $4^{\circ} \mathrm{C}$. Aliquots of the supernatant were transferred to the HPLC system.

The HPLC system was described in the preceding paragraph, with the potentials being set at +450 and $+100 \mathrm{mV}$ at the analytical and the conditioning cells, respectively. The column, a Nova-Pack phenyl column $(3.9 \times 150 \mathrm{~mm})$ and a Sentry Guard Nova-Pack precolumn $(3.9 \times$ $20 \mathrm{~mm}$ ) were purchased from Waters Corporation. The flow rate was 1 $\mathrm{ml} / \mathrm{min}$. The mobile phase consisted of $3 \%$ methanol in $0.1 \mathrm{M} \mathrm{Na}$ phosphate buffer, pH 3, $0.1 \mathrm{~mm} \mathrm{Na} 2 \mathrm{EDTA}$, and $0.5 \mathrm{~mm}$ 1-octane sulfonic acid Na salt (Aldrich, Milwaukee, WI).

\section{Statistics}

NE depletion. The effects of prefrontal NE depletion on tissue levels of DA and NE in the mpFC and in the NAc were analyzed by two-way ANOVA, with factors as follows: lesion (two levels: Sham and NE depleted); and experiment (three levels: behavioral experiment, microdialysis in the NAc, and microdialysis in the mpFC) $(n=85)$. Individual betweengroup comparisons, when appropriate, were performed by post hoc test (Duncan's multiple range test).

Conditioned place preference. For CPP experiments, statistical analyses were performed on preference scores, assessed by calculating the time spent in Amph (Paired) and saline-paired (Unpaired) compartments on the test day minus the time spent in the same compartments on the pretest session. In the case of animals receiving saline pairing with both compartments, the Paired compartment was identified as the first to which they were exposed. Data from CPP experiments $(n=23)$ were analyzed using repeated-measures ANOVA, with one between factor (lesion, two levels: Sham and NE depleted) and one within factor (pairing, two levels: Paired and Unpaired) for both saline- and Amph-treated mice. Locomotor activity was analyzed by three-way ANOVA, with the factors being treatment (two levels: saline and Amph), lesion (two levels: Sham and NE depleted), and day [two levels: first day (first pairing) and last day (last pairing)]. Simple effects were assessed by one-way ANOVA.

Microdialysis. Statistical analyses were performed on raw data (concentrations, pg/20 $\mu \mathrm{l}$ ). The effects of Amph on extracellular NE levels in the mpFC was analyzed by repeated-measures ANOVA with one between factor (treatment, two levels: saline and Amph) and one within factor (minutes, seven levels: $0,20,40,60,80,100$, and 120) $(n=18)$. The effects of prefrontal NE depletion on DA release in the NAc of animals $(n=47)$ challenged with Amph were analyzed by repeated-measures ANOVA, with two between factors (treatment, two levels: saline and Amph; and lesion, two levels: Sham and NE depleted) and one within factor (minutes, seven levels: 0, 20, 40, 60, 80, 100, and 120). The effects of prefrontal NE depletion on NE and DA release in the mpFC of animals $(n=15)$ challenged with Amph were analyzed by repeated-measures ANOVA, with one between factor (lesion, two levels: Sham and NE depleted) and one within factor (minutes, nine levels: $-40,-20,0,20,40$, $60,80,100$, and 120). Simple effects were assessed by one-way ANOVA for each time point. Individual between-group comparisons, when ap- propriate, were performed by post hoc test (Duncan's multiple range test).

\section{Results \\ NE depletion}

Statistical analysis for effects of prefrontal NE depletion on DA and NE tissue levels in the mpFC showed no significant experiment effect and a significant lesion effect $\left(F_{(2,79)}=7.08 ; p<\right.$ $0.0005)$ only for NE values, whereas dopamine levels did not differ significantly. No significant experiment or lesion effect was evident for either NE or DA in the NAc (Table 1).

\section{Conditioned place preference}

The effects of NE depletion in the medial prefrontal cortex on Amph-induced place conditioning are shown in Figure 2. The results showed no preference for either compartment by animals that had experienced saline pairing with both compartments, regardless of the lesion condition (Sham or NE depleted) (Fig. $2 A)$. As for animals from the Amph-treated groups, ANOVA revealed a significant pairing $\times$ lesion interaction $\left(F_{(1,11)}=6.3\right.$; $p<0.05)$. Sham group animals showed a significant preference for the Amph-paired compartment $\left(F_{(1,12)}=7.6 ; p<0.05\right)$, but Amph failed to induce any preference for the drug-paired compartment in the NE-depleted group (Fig. $2 B$ ).

The effects of Amph-induced locomotor activity are shown in Figure 3. ANOVA showed significant lesion $\left(F_{(1,38)}=8.58 ; p<\right.$ $0.01)$ and treatment main effects $\left(F_{(1,38)}=122.2 ; p<0.0005\right)$ and a significant treatment $\times$ day interaction $\left(F_{(1,38)}=17.7 ; p<\right.$ $0.0005)$. Simple effect analyses revealed significant effects of lesion and day only in Amph-treated groups. Amph-treated animals from both Sham and NE-depleted groups showed significantly increased locomotor activity on the last day compared with that exhibited on the first drug pairing. Moreover, betweengroup comparisons showed that Amph-induced locomotor activity in NE-depleted mice was higher than that induced in Sham mice on the last day (Fig. 3B).

\section{Microdialysis}

The effects of Amph on NE release in the mpFC are shown in Figure 4. Statistical analyses revealed a significant treatment $X$ minutes interaction $\left(F_{(1,96)}=9.52 ; p<0.0005\right)$. Simple effect analyses revealed a significant effect of minutes only for Amph and a significant difference between saline and Amph at all time points. Amph produced a clear-cut increase in NE compared with saline along the $120 \mathrm{~min}$ postinjection period, reaching a maximal of $\sim 400 \%$ increase at $40 \mathrm{~min}$ after injection. No significant effect was evident in saline-injected mice.

The effects of prefrontal NE depletion on DA release induced by systemic Amph in the NAc are shown in Figure 5. Statistical analyses revealed a significant treatment $\times$ lesion $\times$ minutes interaction $\left(F_{(1,258)}=5.63 ; p<0.0005\right)$. Simple effect analysis revealed a significant effect of minutes only for Amph and significant difference between saline and Amph. Amph produced a significant increase in DA release in the NAc of Sham mice com-

Table 1. NE and DA tissue levels (ng/gm wet weight) in $\mathrm{mpFC}$ and NAc of sham and NE-depleted mice

\begin{tabular}{llcccc}
\hline & \multicolumn{3}{l}{ Sham } & & NE depleted \\
\cline { 2 - 3 } \cline { 5 - 6 } & NE & DA & & NE & DA \\
\hline mpFC & $701 \pm 28$ & $197 \pm 11$ & & $67 \pm 19^{*}$ & $183 \pm 15$ \\
NAC & $729 \pm 49$ & $13752 \pm 814$ & & $848 \pm 62$ & $12792 \pm 589$ \\
\hline${ }^{*} p<0.0005$. & & & &
\end{tabular}




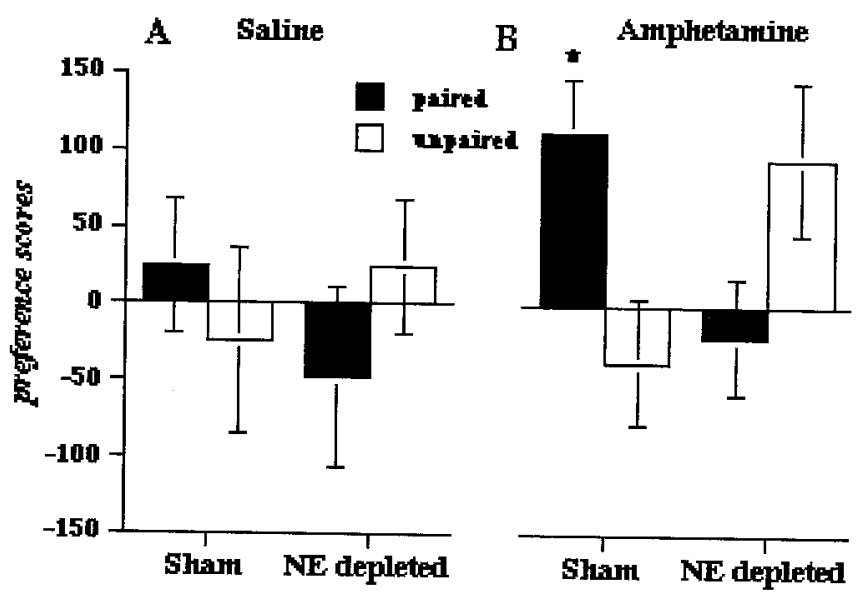

Figure 2. Effects of prefrontal cortical norepinephrine depletion on the preference scores (for details, see Materials and Methods) shown by saline $(A)$ and amphetamine ( $B$ )-treated groups in conditioned place preference test. All data are expressed as mean \pm SE. ${ }^{*} p<0.05$ compared with the unpaired compartment.

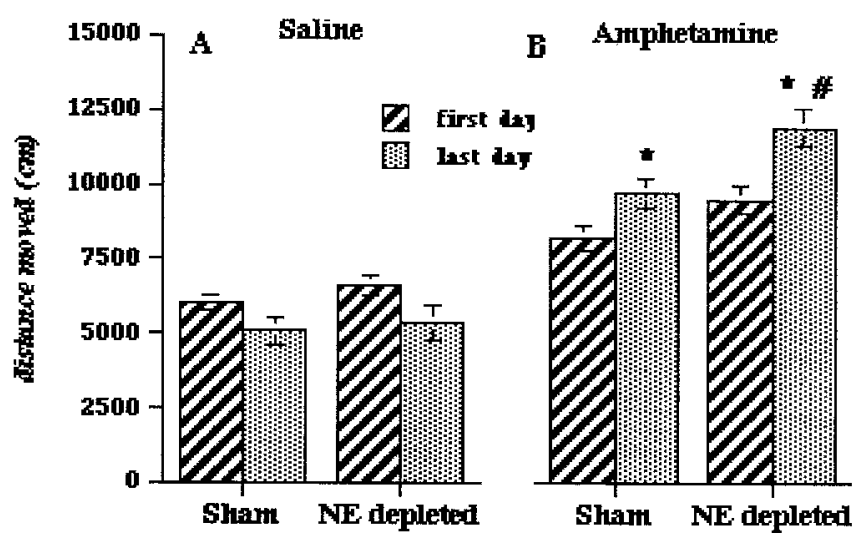

Figure 3. Effects of prefrontal cortical norepinephrine depletion on the locomotor activity induced by saline $(A)$ and amphetamine $(B)$ on the first day (first pairing) or the last day (last pairing) of pairing section. All data are expressed as mean \pm SE. ${ }^{*} p<0.05$ compared with day 1. \#p $<0.05$ compared with the Sham group.

pared with saline over the 100 min postinjection period, reaching a peak value (350\% of basal values) at $40 \mathrm{~min}$ after injection. Moreover, Amph failed to produce increased DA release in the NAc of prefrontal NE-depleted mice. Basal DA outflow did not differ significantly between groups.

To assess whether selective NE depletion in the mpFC affected extracellular NE and DA, we measured the effects of Amph on prefrontal cortical amine outflow. The effects of prefrontal NE depletion on NE and DA release induced by systemic Amph in the mpFC are shown in Figure 6. Statistical analyses revealed a significant lesion $\times$ minutes interaction $\left(F_{(1,104)}=33.72 ; p<\right.$ $0.0005)$ for NE only. Simple effect analyses revealed a significant effect of minutes only for the Sham group and a significant difference between Sham and NE-depleted groups at all time points after Amph injection. No significant differences were evident in basal extracellular NE between Sham and NE-depleted animals. However, no significant changes occurred in NE-depleted cortex after Amph challenge. No significant differences were evident between Sham and NE-depleted animals in both basal and Amph-induced extracellular DA.

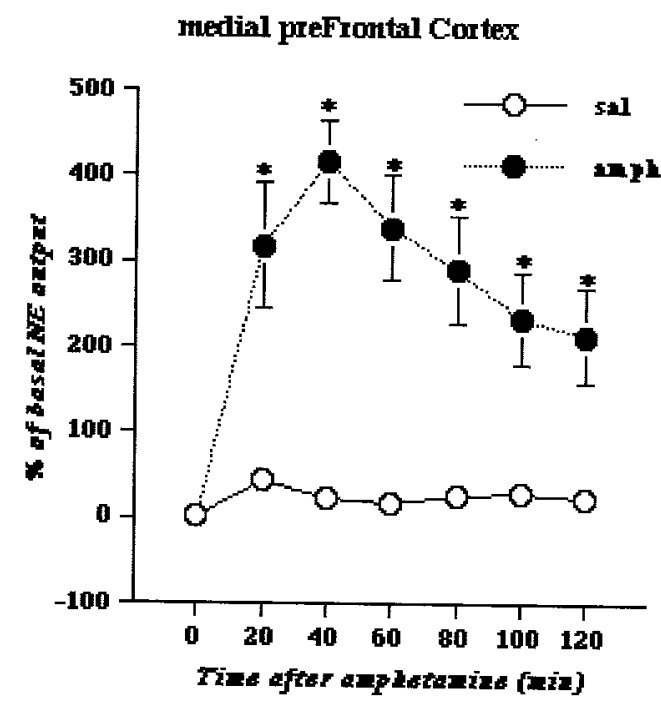

Figure 4. Extracellular norepinephrine in the medial prefrontal cortex of animals receiving saline (sal) or amphetamine $(2.5 \mathrm{mg} / \mathrm{kg}$, i.p.) $(\mathrm{amph})$. Results are expressed as percentage changes (means $\pm \mathrm{SE}$ ) from basal values $(1.16 \pm 0.12 \mathrm{pg} / 20 \mu \mathrm{l})$. Statistical analyses were performed on raw data. ${ }^{*} p<0.01$ compared with saline.
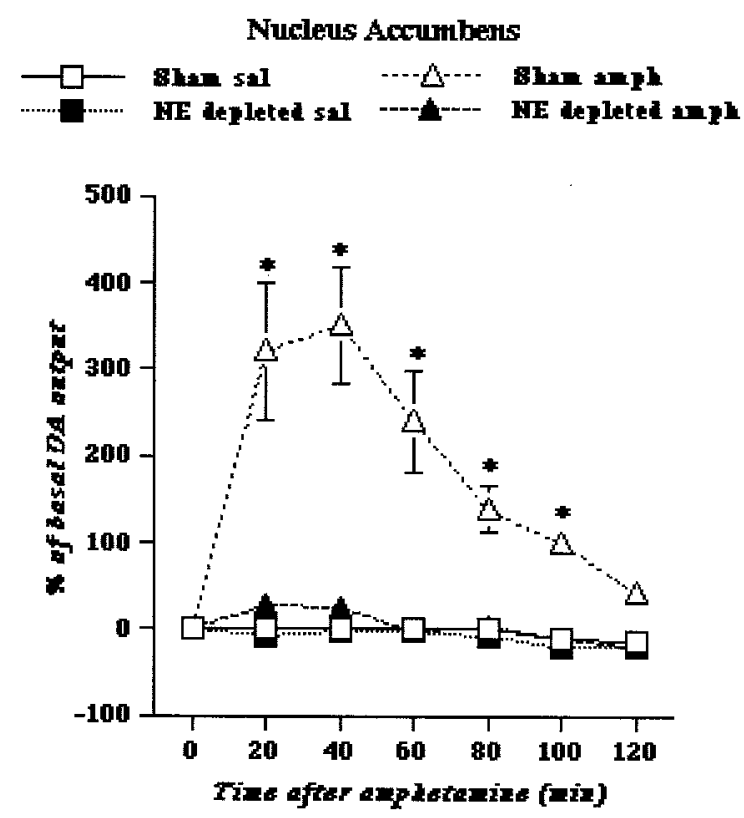

Figure 5. Effects of prefrontal cortical norepinephrine depletion on extracellular dopamine in the nucleus accumbens of animals receiving saline ( $\mathrm{sal}$ ) or amphetamine $(2.5 \mathrm{mg} / \mathrm{kg}$, i.p.) (amph). Results are expressed as percentage changes (means $\pm \mathrm{SE}$ ) from basal values ( $1.30 \pm$ $0.16 \mathrm{pg} / 20 \mu \mathrm{l}$ ). Statistical analyses were performed on raw data. ${ }^{*} p<0.01$ compared with saline.

\section{Discussion}

The first major finding in the present study is that prefrontal NE depletion blocks CPP induced by systemic Amph. In fact, whereas Sham mice exhibited a significant preference for the Amph-paired compartment, no preference was evident in the NE-depleted group. If anything, the latter group displayed a nonsignificant preference for the saline-paired compartment. Preference for the compartment not paired with Amph is attributable to an increase in the time spent in this compartment on the test day. In NE-depleted animals, this possibly indicates a slightly aversive reaction to the drug-paired compartment (Cabib et al., 

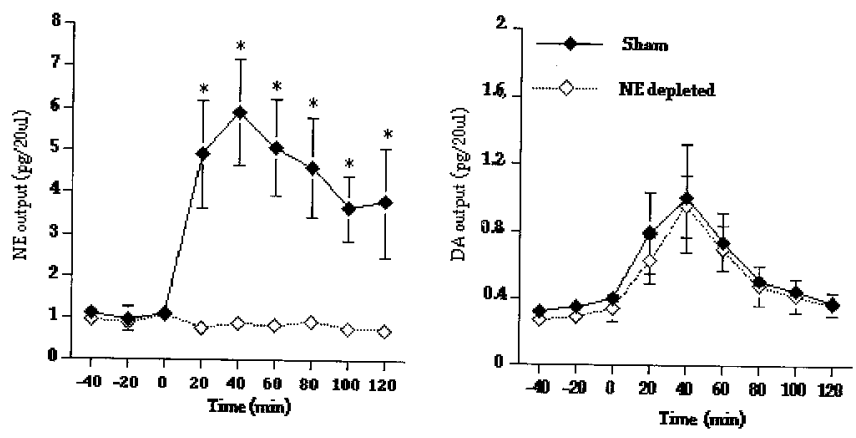

Figure 6. Effects of prefrontal cortical norepinephrine depletion on extracellular norepinephrine and dopamine in the medial prefrontal cortex of animals receiving amphetamine ( 2.5 $\mathrm{mg} / \mathrm{kg}$, i.p.). ${ }^{*} p<0.01$ compared with the Sham group.

1996, 2000). In alternation as well as in addition, the increase in the time spent in the compartment paired previously with vehicle might indicate thorough exploration of an environment perceived as novel because of memory disturbances promoted by pFC NE depletion (Kobayashi et al., 2000; Gibbs and Summers, 2002.). Amph may have facilitated memorization of the drugpaired compartment in NE-depleted mice by its action on a different brain area (Mattay et al., 1996; Hsu et al., 2002) or on a different neurotransmitter (Castellano et al., 1996), leading on the test day to lower exploration of a known and slightly aversive environment. This likelihood warrants future specific experimental testing because it further supports the implication of a selective reduction of the positive reinforcing effects of Amph in the NE-depleted group. Meanwhile, the locomotor activity data offer indirect support to the hypothesis that prefrontal cortical NE depletion selectively affects the positive reinforcing effects of Amph. Indeed the NE-depleted group exhibited clear evidence of behavioral sensitization to the locomotor stimulating effects of Amph on the last day of pairing. It should be pointed out that mice of the C57 strain are characterized by low susceptibility to context-independent and high susceptibility to context-dependent sensitization (for review, see Puglisi-Allegra and Cabib, 1997) a phenomenon that is highly dependent on the ability to associate the context with the drug effects. Thus, mpFC NE depletion does not interfere with associative processes in Amph-treated animals.

The effects of selective NE depletion on the rewarding effects of Amph reported here may appear at odds with previous reports of an absence of any effect of excitotoxic pFC lesions on Amphinduced CPP (Tzschentke and Schmidt 1998a). Nonetheless, this discrepancy is not surprising in view of the conflicting results obtained by different manipulations of $\mathrm{pFC}$ on behavioral responses to the psychostimulant. Thus, although excitotoxic lesions as well as ablation of the $\mathrm{pFC}$ have been reported to promote enhanced locomotor response to Amph (Whishaw et al., 1992; Dalley et al., 1999; Roffman et al., 2000), in some research no effect of pFC lesions on Amph-induced locomotion was found (Burns et al., 1993; Tzschentke and Schmidt, 1998a). Moreover, excitotoxic lesions do not always reproduce the effects of cortical aminergic depletion (Collins et al., 1998). We found that prefrontal NE depletion blocked CPP but did not reduce the motor-activating effects induced by Amph. Our results are in agreement with a number of studies indicating only very weak or no correlation between the motor stimulating and rewardingreinforcing effects of drugs of abuse (for review, see Tzschentke, 1998). However, previous studies have reported that reduced NE transmission prevents the locomotor hyperactivity induced by intra-NAc injection of Amph (Blanc et al., 1994) and reduces the acute locomotor effect of systemic Amph (Drouin et al., 2002a). Moreover, mice lacking the $\alpha 1$-subtype adrenergic receptors have been shown to exhibit lower Amph-induced locomotor activity than the wild-type (Drouin et al., 2002b).

Discrepancies between these previous and the present results may depend on differences in the testing condition. Thus, different from experimental procedures specifically aimed at assessing locomotor effects of psychostimulants (Cabib et al., 2000; Auclair et al., 2002), we did not use prolonged habituation to the testing cages before drug challenge, to avoid the risk of a latent inhibition of association between drug effects and environmental stimuli. Nevertheless, studies using NE depletion indicated preserved locomotor response to Amph (Archer et al., 1986; Geyer et al., 1986; Mohammed et al., 1986). Therefore, it may be that manipulations of NE transmission promote complex effects that depend on the extent and/or selectivity of alterations of cortical functioning.

It is worth noting that we used an original experimental procedure to induce a massive but selective prefrontal NE depletion ( $>90 \%$ ), producing only a weak, nonsignificant reduction in tissue DA levels ( $\sim 7 \%)$, without affecting either DA or NE tissue levels in the NAc. To the best of our knowledge, this is the first report of the effects of a selective neurotoxic lesion of prefrontal NE. Although the NE depletion produced a dramatic decrease in the neurotransmitter tissue levels in the mpFC, basal extracellular NE values in dialysate were not different from those of Sham animals. These results suggest that spared noradrenergic afferents develop a compensatory response that leads to an extracellular NE outflow similar to that of Sham animals, in agreement with previous studies based on nonselective NE depletion (Abercrombie and Zigmond, 1989; Hughes and Stanford, 1998). Whether this compensatory response depends on increased neurotransmitter synthesis or on other mechanisms remains to be ascertained. However, NE depletion abolished the noradrenergic response to Amph challenge in the mpFC, possibly indicating that compensatory response does not allow additional increase in $\mathrm{NE}$ outflow after Amph challenge.

The second important finding of this study is the dramatic reduction of amphetamine-induced DA release in the NAc of prefrontal NE-depleted mice. Thus, in the Sham group, systemic Amph produced a large and significant increase in DA outflow in the NAc ( $\sim 350 \%$ maximal increase), which reached a peak value at $40 \mathrm{~min}$ after injection. Conversely, no significant increase was observable in the NAc of NE-depleted animals, thus pointing to intact noradrenergic transmission within the mpFC being a necessary condition for Amph-stimulated DA release within the NAc. Very recent results showing that Amph fails to increase extracellular DA in the NAc of mice lacking $\alpha 1$ b-adrenergic receptors (Auclair et al., 2002) support this view.

The present results cannot be ascribed to reduced DA or NE tissue levels in the NAc that were not affected by prefrontal cortical NE depletion, thus ruling out that the 6-OHDA diffused to the NAc.

Cortical NE may participate in the Amph-promoted DA release within the NAc through different mechanisms. First, it might activate the excitatory prefronto-cortical projection to the ventral tegmental area (VTA). Indeed, recent results have revealed an NE-dependent excitation of VTA DA neurons in Amph-treated animals (Shi et al., 2000), thus supporting the view that a functional amount of Amph-induced mesoaccumbens DA release is impulse flow dependent (Darracq et al., 1998; Shi et al., 2000; Paladini et al., 2001). Second, it might activate corticoaccumbal glutamatergic projections that stimulate AMPA-kain- 
ate NMDA presynaptic receptors located on DA nerve terminals facilitating release (Darracq et al., 2001). Third, it might facilitate the ionotropic receptor-mediated activation of efferent inhibitory GABAergic neurons. These neurons participate in a double inhibition loop involved in the control of DA cell activity by local GABAergic neurons (Darracq et al., 2001). The marked effect of cortical NE depletion on Amph-induced accumbal DA release in our experiments points to the involvement of all three mechanisms. Thus, elimination of the NE-mediated excitatory input to VTA DA neurons, of the facilitation of DA release by NMDA receptor within the accumbens, and of inhibitory control of GABAergic tonic inhibition of DA cells might have contributed to a strong reduction of the psychostimulant effects on DA release within the accumbens. It is worth noting that NE depletion did not affect prefrontal cortical DA response to Amph, which may contribute to inhibition of accumbal DA release through activation of $D_{1}$ receptors located on cortical glutamatergic efferents (Tassin, 1998). A role of $\mathrm{D}_{4} \mathrm{DA}$ receptors in the effects of $\mathrm{NE}$ has to be taken into account in the light of affinity of NE for this receptor subtype and of their possible involvement in the effects of Amph (Newman-Tancredi et al., 1997; Feldpausch et al., 1998).

Together, the disruption of these various mechanisms by mpFC NE depletion may converge to inhibit the activity of DA neurons. Blockade of DA neurons may reduce Amph-induced reversal of the membrane DA transporter (Darracq et al., 2001), providing an explanation of the virtual absence of Amphinduced mesoaccumbens DA release in prefrontal cortical NEdepleted animals. Finally, the dramatic reduction in Amphinduced mesoaccumbens DA release in NE-depleted mice reinforce the behavioral data, indicating blockade of the reinforcing-rewarding effects of the psychostimulant. Indeed, there is convincing evidence to support a major role of mesoaccumbens DA release in CPP induced by amphetamine (Carr and White, 1986; Olmstead and Franklin, 1996; Schildein et al., 1998).

In conclusion, our results for the first time demonstrate a critical role of prefrontal NE transmission in the mediation of the reinforcing-rewarding effects of amphetamine through modulation of DA release in the NAc.

\section{References}

Abercrombie ED, Zigmond MJ (1989) Partial injury to central noradrenergic neurons: reduction of tissue norepinephrine content is greater than reduction of extracellular norepinephrine measured by microdialysis. J Neurosci 9:4062-4067.

Archer T, Fredriksson A, Jonsson G, Lewander T, Mohammed AK, Ross SB, Soderberg U (1986) Central noradrenaline depletion antagonizes aspects of d-amphetamine-induced hyperactivity in the rat. Psychopharmacology 88:141-146.

Auclair A, Cotecchia S, Glowinski J, Tassin J-P (2002) D-Amphetamine fails to increase extracellular dopamine levels in mice lacking $\alpha$ lb-adrenergic receptors: relationship between functional and nonfunctional dopamine release. J Neurosci 22:9150-9154.

Blanc G, Trovero F, Vezina D, Hervè AM, Glowinski J, Tassin JP (1994) Blockade of prefronto-cortical $\alpha$-1-adrenergic receptors prevents locomotor hyperactivity induced by subcortical D-amphetamine injection. J Neurosci 6:293-298.

Bubser M, Schmidt WJ (1990) 6-Hydroxydopamine lesion of the rat prefrontal cortex increases locomotor activity, impairs acquisition of delayed alternation tasks, but does not affect un-interrupted tasks in the radial maze. Behav Brain Res 37:157-168.

Burns LH, Robbins TW, Everitt BJ (1993) Differential effects of excitotoxic lesions of the basolateral amygdala, ventral subiculum and medial prefrontal cortex on responding with conditioned reinforcement and locomotor activity potentiated by intra-accumbens infusions of D-amphetamine. Behav Brain Res 55:167-183.
Cabib S, Puglisi-Allegra S, Genua C, Simon H, Le Moal M, Piazza PV (1996) Dose-dependent aversive and rewarding effects of amphetamine as revealed by a new place conditioning apparatus. Psychopharmacology 125:92-96.

Cabib S, Orsini C, Le Moal M, Piazza PV (2000) Abolition and reversal of strain differences in behavioral responses to drug of abuse after a brief experience. Science 289:463-465.

Carr GD, White NM (1986) Anatomical dissociation of amphetamine's rewarding and aversive effects: an intracranial microinjection study. Psychopharmacology 89:340-346.

Carter CJ, Pycock CJ (1980) Behavioural and biochemical effects of dopamine and noradrenaline depletion within the medial prefrontal cortex of the rat. Brain Res 192:163-176.

Castellano C, Cabib S, Puglisi-Allegra S (1996) Psychopharmacology of memory modulation: evidence for multiple interaction among neurotransmitters and hormones. Behav Brain Res 77:1-21.

Collins P, Roberts AC, Dias R, Everitt BJ, Robbins TW (1998) Perseveration and strategy in a novel spatial self-ordered sequencing task for nonhuman primates. Effects of excitotoxic lesions and dopamine depletions of the prefrontal cortex. J Cognit Neurosci 10:332-354.

Dalley JW, Thomas KL, Howes SR, Tsai TH, Aparicio-Legarza MI, Reynolds GP, Everitt BJ, Robbins TW (1999) Effects of excitotoxic lesions of the rat prefrontal cortex on CREB regulation and presynaptic markers of dopamine and amino acid function in the nucleus accumbens. Eur J Neurosci 11:1265-1274.

Darracq L, Blanc G, Glowinski J, Tassin JP (1998) Importance of the noradrenaline-dopamine coupling in the locomotor activating effects of D-amphetamine. J Neurosci 18:2729-2739.

Darracq L, Drouin C, Blanc G, Glowinski J, Tassin JP (2001) Stimulation of metabotropic but not ionotropic glutamatergic receptors in the nucleus accumbens is required for the D-amphetamine-induced release of functional dopamine. Neuroscience 103:395-403.

Di Chiara G (1995) The role of dopamine in drug abuse from the perspective of its role in motivation. Drug Alcohol Depend 38:95-137.

Di Chiara G, Imperato A (1988) Drugs abused by humans preferentially increase synaptic dopamine concentrations in the mesolimbic system of freely moving rats. Proc Natl Acad Sci USA 85:5274-5278.

Dickinson SL, Gadie B, Tulloch IF (1988) Alpha 1- and alpha 2-adrenoreceptor antagonists differentially influence locomotor and stereotyped behaviour induced by $d$-amphetamine and apomorphine in the rat. Psychopharmacology 96:521-527.

Drouin C, Blanc G, Villègier AS, Glowinski J, Tassin JP (2002a) Critical role of alpha-1b-adrenergic receptors in acute and sensitized locomotor effects of D-amphetamine, cocaine, and GBR 12783: influence of preexposure conditions and pharmacological characteristics. Synapse 43:51-61.

Drouin C, Darracq L, Trovero F, Blanc G, Glowinski J, Cotecchia S, Tassin J-P (2002b) $\alpha$-1b-Adrenergic receptors control locomotor and rewarding effects of psychostimulants and opiates. J Neurosci 22: 2873-2884.

Feldpausch DL, Needham LM, Stone MP, Althaus JS, Yamamoto BK, Svensson KA, Merchant KM (1998) The role of dopamine D4 receptor in the induction of behavioral sensitization to amphetamine and accompanying biochemical and molecular adaptation. J Pharmacol Exp Ther 286:497-508.

Franklin KBJ, Paxinos G (1998) The mouse brain in stereotaxic coordinates. San Diego: Academic.

Geyer MA, Masten VL, Segal DS (1986) Behavioral effects of xylamineinduced depletions of brain norepinephrine: interaction with amphetamine. Behav Brain Res 21:55-64.

Gibbs M, Summers R (2002) Role of adrenoceptor subtypes in memory consolidation. Prog Neurobiol 67:345-391.

Hsu EH, Schroeder JP, Packard MG (2002) The amygdala mediates memory consolidation for an amphetamine conditioned place preference. Behav Brain Res 129:93-100.

Hughes ZA, Stanford SC (1998) A partial noradrenergic lesion induced by DSP-4 increases extracellularnoradrenaline concentration in rat frontal cortex: a microdialysis study in vivo. Psychopharmacology 36:299-303.

Kobayashi K, Noda Y, Matsushita N, Nishii K, Sawada H, Nagatsu T, Nakahara D, Fukabori R, Yasoshima Y, Yamamoto T, Miura M, Kano M, Mamiya T, Miyamoto Y, Nabeshima T (2000) Modest neuropsychological deficits caused by reduced noradrenaline metabolism in mice heterozygous for a mutated tyrosine hydroxylase gene. J Neurosci 20:2418-2426. 
Koob GF, Sanna PP, Bloom FE (1998) Neuroscience of addiction. Neuron 21:467-476.

Kuczenski R, Segal DS (1997) Effects of methylphenidate on extracellular dopamine, serotonin, and norepinephrine: comparison with amphetamine. J Neurochem 68:2032-2037.

Mattay VS, Berman KF, Ostrem JL, Esposito G, Van Horn JD, Bigelow LB, Weinberger DR (1996) Dextroamphetamine enhances "neural networkspecific" physiological signals: a positron-emission tomography rCBF study. J Neurosci 16:4816-4822.

Mohammed AK, Danysz W, Ogren SO, Archer T (1986) Central noradrenaline depletion attenuates amphetamine-induced locomotor behavior. Neurosci Lett 64:139-144.

Newman-Tancredi A, Audinot-Bouchez V, Gobert A, Millan MJ (1997) Noradrenaline and adrenaline are high affinity agonists at dopamine D4 receptors. Eur J Pharmacol 319:379-383.

Olmstead MC, Franklin KB (1996) Differential effects of ventral striatal lesions on the conditioned place preference induced by morphine and amphetamine. Neuroscience 71:701-708.

Paladini CA, Fiorillo CD, Morikawa H, Williams JT (2001) Amphetamine selectively blocks inhibitory glutamate transmission in dopamine neurons. Nat Neurosci 4:275-280.

Pan WH, Sung JC, Fuh SM (1996) Locally application of amphetamine into the ventral tegmental area enhances dopamine release in the nucleus accumbens and the medial prefrontal cortex through noradrenergic neurotransmission. J Pharmacol Exp Ther 278:725-731.

Pontieri FE, Tanda G, Di Chiara G (1995) Intravenous cocaine, morphine, and amphetamine preferentially increase extracellular dopamine in the "shell" as compared with the "core" of the rat nucleus accumbens. Proc Natl Acad Sci USA 92:12304-12308.

Puglisi-Allegra S, Cabib S (1997) Psychopharmacology of dopamine: The contribution of comparative studies in inbred strains of mice. Prog Neurobiol 51:637-661.

Robbins TW, Everitt BJ (1999) Drug addiction: dab habits add up. Nature 398:567-570.

Robinson TE, Berridge KC (2001) Incentive-sensitization and addiction. Addiction 96:103-114.

Roffman JL, Lipska BK, Bertolino A, Van Gelderen P, Olson AW, Khaing ZZ, Weinberger DR (2000) Local and downstream effects of excitotoxic lesions in the rat medial prefrontal cortex on in vivo $1 \mathrm{H}-\mathrm{MRS}$ signals. Neuropsychopharmacology 22:430-439.

Rothman RB, Baumann MH, Dersch CM, Romero DV, Rice KC, Carroll FI, Partilla JS (2001) Amphetamine-type central nervous system stimulants release norepinephrine more potently than they release dopamine and serotonin. Synapse 39:32-41.

Schildein S, Agmo S, Huston JP, Schwarting RKW (1998) Intraaccumbens injections of substance $\mathrm{P}$, morphine and amphetamine: effects on conditioned place preference and behavioral activity. Brain Res 790:185-194.
Shi WX, Pun CL, Zhang XX, Jones MD, Bunney BS (2000) Dual effects of D-amphetamine on dopamine neurons mediated by dopamine and nondopamine receptors. J Neurosci $203504-3511$.

Snoddy AM, Tessel RE (1985) Prazosin: effect on psychomotor-stimulant cues and locomotor activity in mice. Eur J Pharmacol 116:221-228.

Spink AJ, Tegelenbosch RA, Buma MO, Noldus LP (2001) The EthoVision video tracking system: a tool for behavioral phenotyping of transgenic mice. Physiol Behav 73:731-744.

Tassin J-P (1998) Norepinephrine-dopamine interactions in the prefrontal cortex and the ventral tegmental area: relevance to mental diseases. Adv Pharmacol 42: 712-716.

Tzschentke TM (1998) Measuring reward with the conditioned place preference paradigm: a comprehensive review of drug effects, recent progress and new issues. Prog Neurobiol 56:613-672.

Tzschentke TM, Schmidt WJ (1998a) Discrete quinolinic acid lesions of the rat prelimbic medial prefrontal cortex affect cocaine- and MK-801-, but not morphine- and amphetamine-induced reward and psychomotor activation as measured with place preference conditioning paradigm. Behav Brain Res 97:115-127.

Tzschentke TM, Schmidt WJ (1998b) Effects of discrete quinolinic acid lesions of the rat infralimbic medial prefrontal cortex on drug-induced conditioned place preference. Behav Pharmacol 9 [Suppl 1]:S87.

Ventura R, Cabib S, Puglisi-Allegra S (2001) Opposite genotype-dependent mesocorticolimbic dopamine response to stress. Neuroscience 104:627-631.

Vezina P (1993) Amphetamine injected into the ventral tegmental area sensitizes the nucleus accumbens dopaminergic response to systemic amphetamine: an in vivo microdialysis study in the rat. Brain Res 605:332-337.

Volkow ND, Fowler JS (2000) Addiction, a disease of compulsion and drive: involvement of the orbitrofrontal cortex. Cereb Cortex 10:318-325.

Weiss F, Hurd YL, Ungerstedt U, Markou A, Plotsky PM, Koob GF (1992) Neurochemical correlates of cocaine and ethanol self-administration. Ann NY Acad Sci 654:220-241.

Westerink BHC, Enrico P, Feimann J, De Vries JB (1998) The pharmacology of mesocortical dopamine neurons. A dual-probe microdialysis study in the ventral tegmental area and prefrontal cortex of the rat brain. J Pharmacol Exp Ther 285:143-154.

Whishaw IQ, Fiorino D, Mittleman G, Castaneda E (1992) Do forebrain structures compete for behavioral expression? Evidence from amphetamine-induced behavior, microdialysis, and caudate-accumbens lesions in medial frontal cortex damaged rats. Brain Res 576:1-11.

Wise RA, Rompre PP (1989) Brain dopamine and reward. Annu Rev Psychol 40:194-225.

Xu F, Gainetdinov RR, Wetsel WC, Jones SR, Bohn LM, Miller GW, Wang YM, Caron MG (2000) Mice lacking the norepinephrine transporter are supersensitive to psychostimulants. Nat Neurosci 3:465-471. 